\title{
In-situ TEM on Pd at 0-1 Bar Hydrogen Pressure and 20-300 ${ }^{\circ} \mathrm{C}$
}

T. Yokosawa, ${ }^{*+}$ T. Alan, ${ }^{* *}$ G. Pandraud, ${ }^{* *}$ B. Dam,*** and H.W. Zandbergen*

* Kavli Institute of NanoScience, HREM, Delft University of Technology, Delft, The Netherlands

** DIMES-ECTM, Delft University of Technology, Delft, The Netherlands

*** ChemE, Delft University of Technology, Delft, The Netherlands

+ t.yokosawa@tudelft.nl

In order to understand loading and unloading mechanism of hydrogen storage materials, it is important to study this process at the atomic level. In-situ transmission electron microscopy (TEM) at application pressures (1-10 bar) of hydrogen gas is expected to provide the most direct information. Such a pressure in combination with the high vacuum of the TEM can be achieved by the use of a nanoreactor, which is a novel way to perform in situ TEM with atomic resolution at ambient gas pressure [1], thus allowing the study of all kinds of chemical reactions under industrially relevant gas pressures.

Here, we report an in-situ TEM study of the hydrogenation of nanoparticles of palladium using a nanoreactor operated at hydrogen gas pressures of 0-200 mbar and temperatures between 20 and 300 C. The MEMS based nanoreactor is composed of two Si chips with square-shaped $1 \mu \mathrm{m}$ thick SiN membranes which contain very thin SiN windows (about $20 \mathrm{~nm}$ thick) (Figures 1(a) and 1(b)). One of the chips contains a Pt heater and has an inlet and outlet for the gas. Pd was deposited as a $10 \mathrm{~nm}$ thick thin film on the other chip that did not contain the heater. This Pd film was annealed at higher temperature, which resulted in mainly (111)-oriented grains. The specimen holder includes O-rings to obtain leak tight connections between the holder and the nanoreactor (Figure 1(b)). We have developed a gas supply system to allow any gas pressure between 0 and 10 bar to pass via the TEM holder through the nanoreactor. Various measurements were taken to reduce contamination originating from hydrocarbons, which may for instance originate from the gas container.

We have performed in-situ TEM (using a Philips CM30T) of the hydrogenation of palladium by varying the hydrogen gas pressure and/or by changing the temperature. A sequence of diffraction patterns taken during an increase in the hydrogen pressure is shown in Figure 2. In general, within one to two seconds all Pd particles are hydrogenated, but within this time frame one can observe that some particles are hydrogenated quicker than others. Upon loading with hydrogen at 200 mbar the lattice expands with about $3.4 \%$, which is consistent with bulk values (3.3\% change) [2]. Both heating at high temperature and lowering the pressure resulted in dehydrogenating.

In conclusion, we succeeded to hydrogenate and dehydrogenate nanocrystalline Palladium and observe the lattice changes in diffraction mode. We are currently trying to perform in-situ TEM for other hydrogen storage materials, such as $\mathrm{LaNi}_{5}$, which requires a higher pressure $(\sim 4$ bar $)$ of hydrogen gas for hydrogenation.

\section{References}

[1] J.F. Creemer et al., Ultramicroscopy, 108 (2008) 993.

[2] A.J. Maeland, J. Phys. Chem., 65 (1961) 1270.

[3] The authors thank ACTS for financial support. 

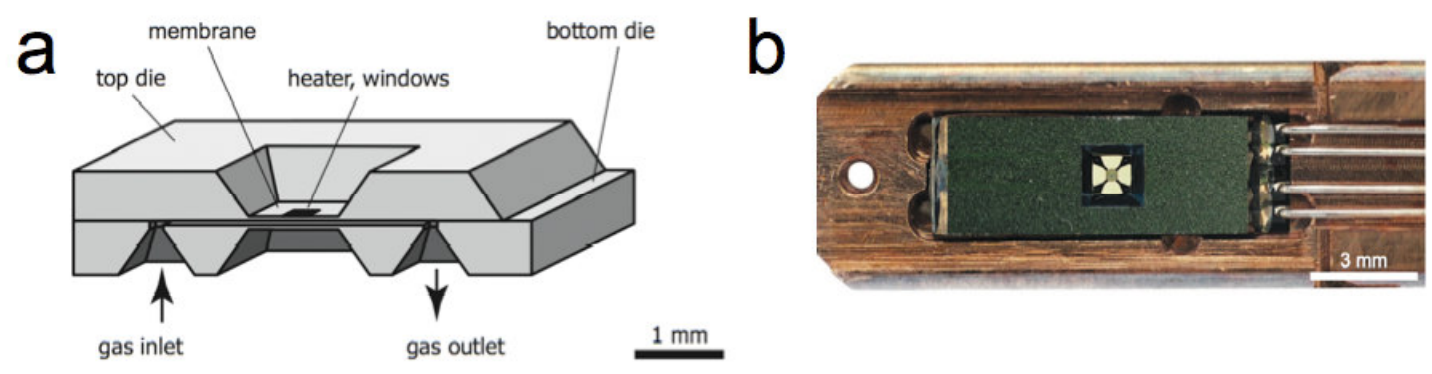

FIG. 1. Illustration of the nanoreactor set-up. a) Schematic cross section of the nanoreactor. b) Optical image of the TEM holder with the integrated nanoreactor and the four electrical probe contacts. The lid on top of the heater to clamp the nanoreactor to the holder thus sealing the inlet and outlet with O-rings is removed.
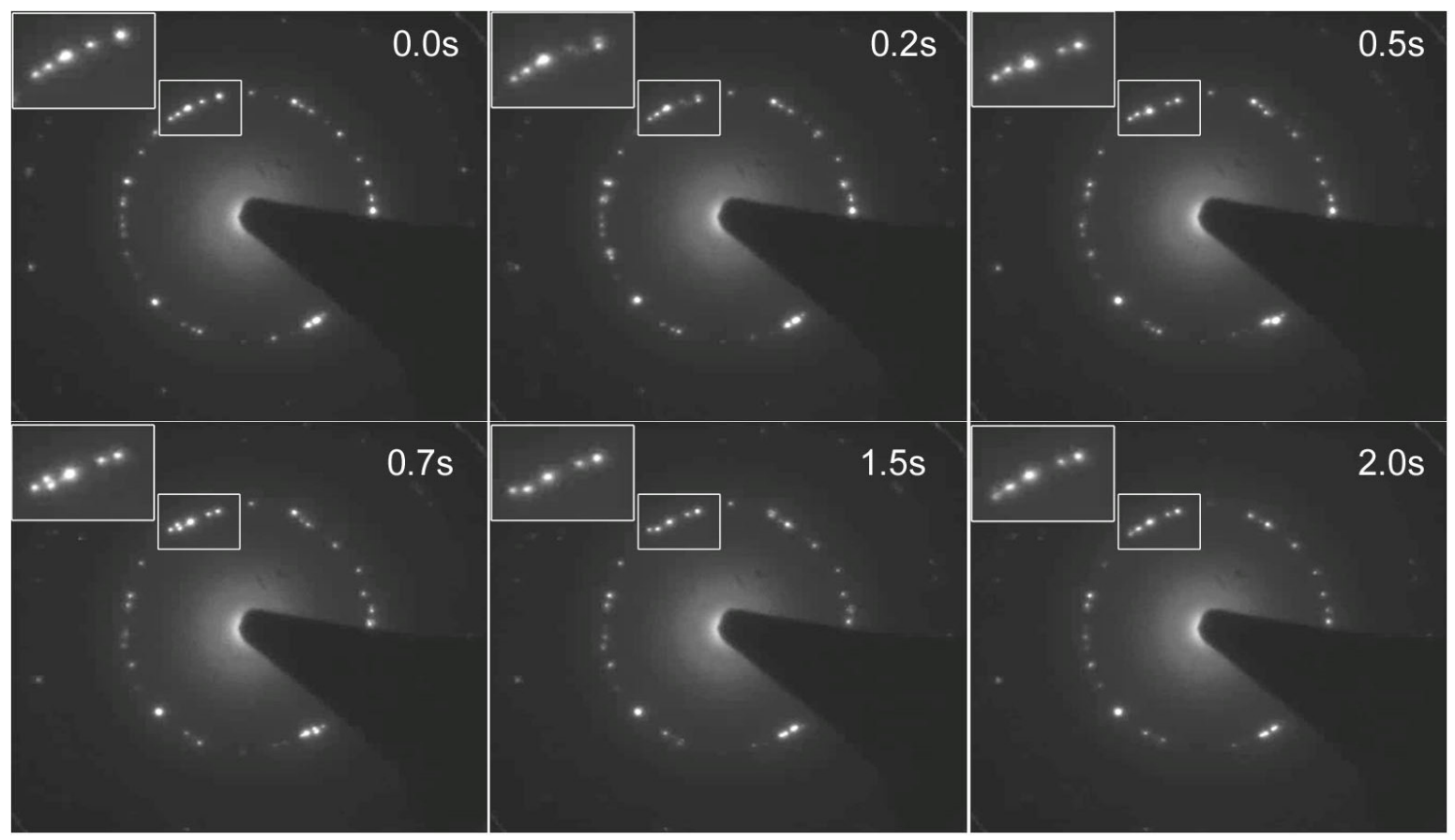

FIG. 2. Frames from a movie taken in SAED mode of the hydrogen loading of palladium during an increase in the hydrogen pressure. One can see that the hydrogen loading occurs within two seconds and that within this time some particles transform faster than others. 